\title{
EFECTO DE LAS ESTRATEGIAS DE INNOVACIÓN EN EL ÉXITO DE LAS EMPRESAS
}

EFFECT OF INNOVATION STRATEGIES ON BUSINESS SUCCESS

\author{
Felipe Alarcón Vásquez, Mgtr. \\ https://orcid.org/0000-0002-7832-3278 \\ Future Consuting, Arequipa, Perú. \\ felipe.alarcon@pucp.edu.pe \\ Santiago Leal Paredes, Mgtr. \\ https://orcid.org/0000-0002-8448-7201 \\ Instituto Superior Tecnológico Cotacachi, Cotacachi, Ecuador. \\ marlonsantiagoleal@hotmail.com \\ Jorge Flores Pérez, Mgtr. \\ https://orcid.org/0000-0002-1140-8074 \\ Universidad Tecnológica del Perú, Arequipa, Perú. \\ flores.jorge@pucp.pe
}

\section{ARTÍCULO DE REFLEXIÓN}

Recibido: 19 de abril de 2021

Aceptado: 6 de mayo de 2021

\section{RESUMEN}

La innovación es el núcleo de las estrategias de negocios actuales, las empresas son cada vez más globales y competitivas, obligando a las empresas a innovar en productos y servicios. Las estrategias de innovación forman parte de la empresa para llegar al éxito y lo que obliga a las organizaciones a innovar para no salir del mercado. El propósito de la presente investigación es analizar y realizar una reflexión sobre el efecto de las estrategias de innovación en el éxito de las empresas. El objetivo es conocer la tendencia y el impacto que tendrá cada estrategia de innovación. El estudio realizó una amplia revisión de literatura permitiendo analizar las estrategias de innovación con relación al éxito. Las conclusiones están vinculadas a cómo la investigación en innovación genera valor para impulsar el éxito, además de visualizar que tipo de estrategias de innovación utilizar en el área requerida y el tiempo adecuado.

Palabras claves: estrategias de innovación, éxito de las empresas, empresas, innovación.

\section{ABSTRACT}

Innovation is the core of current business strategies, companies are increasingly global and competitive, forcing companies to innovate in products and services. Innovation strategies are part of the company to achieve success and what forces organizations to innovate in order 
not to leave the market. The purpose of this research is to analyze and reflect on the effect of innovation strategies on the success of companies. The objective is to know the trend and the impact that each innovation strategy will have. The study carried out an extensive literature review which allowed analyzing the innovation strategies in relation to success. The conclusions are linked to how innovation research generates value to drive success, in addition to visualizing what type of innovation strategies to use in the required area and the appropriate time.

Keywords: innovation strategies, business success, companies, innovation.

\section{INTRODUCCIÓN}

Las empresas a través del tiempo, siempre han tenido que generar nuevas estrategias que sean creativas e innovadoras, para volverse más competitivas en los mercados, es así que las empresas buscan ser "orgánicas" es decir que tienen un buen equilibrio entre las innovaciones tecnológicas (producto y proceso) y no tecnológicas (marketing y organización) propuestos por Damanpour y Evan ( citado por Rajapathirana \& Hui, 2018) ya que disfrutan del "Modelo de innovación de doble núcleo", donde se combina la innovación tecnológica de abajo hacia arriba con innovación no tecnológica de arriba hacia abajo y producir un rendimiento de empresa más alto y amplificado propuesto por Daft (citado por Rajapathirana \& Hui, 2018). Las combinaciones innovadoras que componen estos IS, resultan que tienen un papel moderador de procesos e innovaciones organizacionales en el efecto de creatividad de producto en su rendimiento. Finalmente, los resultados pueden atraer la atención de la innovación como un foco superación y mejora dentro de la organización (Rajapathirana \& Hui, 2018)

Las estrategias de innovación de productos, como la introducción de nuevos productos y servicios, así como la organización en la innovación afecta positiva y significativamente el desempeño financiero de las empresas en la industria bancaria como en el caso de Kenia. Este estudio estableció que los datos de la base electrónica de clientes, centros de llamadas, préstamos computarizados, respuesta de voz automatizada, sistemas automatizados de conciliación de cheques, préstamo centralizado sistema de aplicación y comercio electrónico de acciones, afectan positivamente (Muigai \& Gitau, 2018).

La innovación no es sólo una cuestión de rentabilidad, sino también de supervivencia, además la decisión de innovar va a depender de la evaluación del costo - beneficio. La presente investigación tiene como propósito analizar el efecto de las estrategias de innovación en el éxito de las empresas. También elegir los tipos de innovación que son más convenientes de acuerdo con la necesidad y los objetivos planteados. De acuerdo con la cuarta edición del Manual de Oslo 2018, la innovación es un producto o proceso, o 
combinación de ambos, nuevo o mejorado. Por lo tanto; las empresas deben tener las capacidades de innovar, teniendo en cuenta los factores externos que influyen en la innovación. También se presenta un acercamiento tipo object-based a la medición, que es el foco complementario en las innovaciones más importantes (o cambios) de la firma y al uso de datos, orientados a los indicadores de tipo de innovación y análisis usando datos de innovación.

La literatura destaca que la cultura organizativa puede estimular la innovación, pero también obstaculizarla, en función de los valores y de los comportamientos que potencie (Naranjo, Jiménez, \& Sanz, 2012). Se ha encontrado evidencia de que la cultura incide en la innovación de la empresa y la innovación está asociada de forma positiva a la cultura adhocrática. Hallazgos de investigaciones respaldaron que la compañía con mayores capacidades de innovación ha influido positiva y muy fuertemente. De esta manera, ayuda a mejorar la capacidad de innovación que es fundamental para las compañías de seguros para definir innovaciones exitosas (Rajapathirana \& Hui, 2018)

\section{REVISIÓN TEÓRICA}

\section{Estrategias de Innovación}

Las empresas son heterogéneas en su elección cuando se trata de estrategia de innovación. Seis estrategias representan el $70 \%$ de las opciones elegidas y son: introducción de producto puro, proceso puro, marketing puro, organización pura, tanto producto como proceso, y finalmente introduciendo todo tipo de innovación al mismo tiempo. Usando el modelo Multinomial Logit, en varias opciones de estrategias de innovación de las empresas. Centrándonos en las estrategias de innovación más utilizadas, encontraron las variables explicativas más significativas para innovación de producto. (Karlsson \& Tavassoli, 2016)

De acuerdo con Kim, Ondracek, Koseoglu, \& Barca (2017) las empresas en la actualidad están aplicando estrategias de innovación empíricas o ineficientes para cada solución. Por lo tanto, las empresas necesitan conocer o implementar más tipos de innovación de forma secuencial para alcanzar el éxito. De acuerdo con Pisano (2015) la innovación radical es el polo opuesto de la innovación incremental. El reto aquí es puramente tecnológico, la aparición de la ingeniería genética y le da un enfoque para el descubrimiento de fármacos y drogas, apoyándose en la biotecnología, además apostaron por la I+D con productos de alto margen.

En cambio, la innovación abierta juega un papel clave en las economías desarrolladas durante la última década. "Habrá nuevas tendencias tecnológicas que impulsarán la innovación, desde blockchain a la digitalización de la edición genómica". (Bogers, Chesbrough, \& Moedas, 2018 p.11) 
Para Hacklin, Björkdahl, \& Wallin (2018) la innovación abierta permite que una empresa obtenga información de afuera hacia adentro, y viceversa, para lograr mejores relaciones y un mejor desempeño de innovación en todas las partes involucradas en el entorno empresarial. La conclusión es que la implementación de estrategias de innovación abierta ayuda a las empresas a centrarse en sus puntos fuertes y los impulsa, para que se adapten mejor a las demandas del mercado.

Para Padayachee, Matthee \& Merwe (2017) las tecnologías disruptivas están cambiando las reglas de competencia. Los Chief Information Officer (CIO) son responsables de la toma de decisiones deben maniobrar tecnologías emergentes que pueden dar como resultado la innovación del modelo comercial y brindar a las empresas la capacidad de competir en mundo, por lo que:

Los CIO están de acuerdo en que podría haber un valor significativo en la utilización de nuevas tecnologías para crear una ventaja competitiva en un mundo ágil. Investiga los factores críticos de éxito que guían la toma de decisiones tecnológicas en un entorno empresarial ágil, además proporciona un marco para que ayuden a decidir sobre estrategias tecnológicas para respaldar la transformación empresarial que permita a sus organizaciones competir en un entorno tecnológico disruptivo (p. 43).

De acuerdo con Pisano (2015) indicó que la innovación incremental es "dominar el arte de la innovación del modelo de negocio. En innovación, las empresas tienen la posibilidad de elegir qué parte de sus esfuerzos para centrarse en la innovación tecnológica y la cantidad a invertir en la innovación del modelo de negocio" (p. 7), contribuyendo así a la formación de empresas.

De acuerdo con Fernández \& Peña (2009) indicaron que "la importancia de tres de las cinco dimensiones de la estrategia de innovación que fueron consideradas. Con excepción de las fuentes externas, la innovación en producto y postura tecnológica, el resto de los componentes de la estrategia de innovación se relacionan positivamente" (p. 90), contribuyendo así a explicar el comportamiento de estos. La Innovación Disruptiva, se refiere a cómo puede un producto o servicio que en sus orígenes nace como algo residual o como una simple aplicación sin muchos seguidores o usuarios convertirse en poco tiempo en el producto o servicio líder del mercado (Clayton M, 1997) La Innovación inversa creada por dos pensadores de estrategia considerados los más importantes de la actualidad, son Govindarajan y Trimble (citado por Martínez \& Gelves 2017) pusieron en conocimiento un nuevo fenómeno que será el motor de un nuevo modelo de crecimiento mundial para las siguientes dos décadas. 
Para Pisano (2014) la innovación de rutina es una estrategia de innovación que significa comprender y aprovechar las fortalezas distintivas existentes para generar y capturar valor. Ver su repertorio de habilidades de I + D, propiedad intelectual, capacidades operativas, relaciones, canales de distribución y marca pueden proteger y ampliar el valor de la innovación. Para Jiménez \& Sanz (2012) según los estudios realizados, las estrategias innovadoras son más relevantes que las estrategias de imitación, aunque presentaron mayor nivel de riesgo, incertidumbre e inversión. A la vez, estas estrategias de tipo innovador son más capaces de desarrollar innovaciones de tipo incremental que las empresas que persiguen estrategias de imitación.

De acuerdo con Solano, Martelo \& Acevedo (2018) los indicadores de medida en innovación fueron: promover la innovación tecnológica y desarrollo de metodologías para mantener la sostenibilidad en el mercado. Estos resultados están relacionados con la declaración que está dentro del estudio, quien expresa que la innovación tecnológica juega un papel fundamental hacia el futuro sostenible de las organizaciones. El análisis dentro del entorno de producción de las empresas permite conocer sus características y acciones para el desarrollo de nuevas estrategias de innovación. Además, se muestra que la adopción de tecnologías en la innovación de procesos organizacionales facilita la incorporación de nuevas formas de trabajo.

Para entender el proceso y de cómo ha ido evolucionando la palabra innovación, se presenta algunos conceptos de autores: Según Schumpeter (2012) la innovación es la imposición de una novedad técnica u organizacional en el proceso de producción y no simplemente el correspondiente invento. Para Drucker (2002) lanzó la idea de que las empresas ya no compiten con productos sino con modelos empresariales. Dichos modelos nacen de la innovación y cuanto más intensa es esta, tanto más competitivos son.

La innovación ha sido un factor decisivo del éxito de todas las empresas de servicios, por lo que no puede seguir considerándose ajeno a las empresas y deben considerarlo dentro de su estrategia. Con relación al costo - beneficio las empresas con mayor capital son las que tienen la facilidad de innovar más que las de poco capital, los bancos y las universidades son las instituciones más innovadoras. La liquidez un factor importantísimo para tomar la decisión, porque algunas empresas deciden no invertir en innovación porque el retorno es a largo plazo, y las empresas miran a corto y mediano por la supervivencia.

Por último, para la OECD/Eurostat, (2018) en el Manual del Oslo 2018 la innovación es un producto o proceso nuevo o mejorado (o una combinación de ellos) que difiere significativamente de los productos o procesos anteriores de la unidad y que se ha puesto a 
disposición de los usuarios potenciales (producto) o se ha puesto en uso por la unidad (proceso).

\section{Éxito de las empresas}

Para Campos \& Sanchis (2015) indicaron que "el éxito de una organización depende, de diferentes factores de contingencia o parámetros estructurales como el tamaño o la antigüedad, entre otros, incluso en organizaciones donde en gran medida se sacrifica una parte de la rentabilidad económica, social o de sus socios" (p. 187), contribuyendo así a una labor social importante. De acuerdo con Oliver \& Stezano (2017) concluyeron que:

La dinámica latinoamericana de producción científica sobre la innovación, en términos globales, se observa que en Latinoamérica ha habido un importante crecimiento sobre la investigación en temas vinculados a la innovación. Según Merigó et al (2016) el líder de producción científica a nivel mundial es Estados Unidos, Reino Unido, países de Europa occidental, Europa del Este, Asia, América Latina y África, respectivamente. Los resultados de este trabajo deben ser así interpretados conforme a estas marcadas diferencias inter-regionales de volumen de producción científica sobre innovación, pero especialmente considerando la creciente relevancia que va adquiriendo este tema en América Latina. (p.76)

Para Fernández \& Peña (2009) en las empresas influye un marco de condiciones comunes para todas, dentro del cual, cada unidad empresarial alcanza niveles de competitividad muy diferentes, de la forma en que se relaciona con un conjunto de factores. Buena parte de los cuales están relacionados directa o indirectamente con la innovación. El desarrollo de estrategias de innovación es de gran importancia para la obtención de ventajas competitivas, además que se relacionan positivamente.

\section{MATERIALES Y MÉTODOS}

Los resultados obtenidos a través del estudio documental revelaron que el gerente debe desarrollar un comportamiento innovador como fuente del cambio, según Vallarino (citado por Chirinos, 2016) y explicó que consta de siete variables: el comportamiento innovador, el trabajo desafiante, la autonomía, la importancia estratégica, el ambiente favorable, la relación con el medio, la diferenciación y la variación de la demanda. A fin de lograr un alto desempeño, sustentado en el desarrollo científico y humano, sin perder de vista las necesidades de su entorno. 
Las empresas más exitosas se caracterizan por tener más desarrollados los sistemas de control de gestión, utilizar una tecnología de sus procesos más avanzados, certificar la calidad de sus productos o servicios, y aplicar métodos de gestión de recursos humanos más avanzados (Rocca, García, \& Duréndez, 2016).

El tamaño de la pyme y la experiencia del gerente tienen una relación positiva con el éxito competitivo. Por lo tanto; las pymes exitosas son las que combinan más apropiadamente la gestión de sus recursos humanos y disponen de sistemas de control de gestión en su organización más desarrollados.

Franco, Mero, \& Mendoza (2018) analizaron las estrategias de innovación tecnológica en el sistema financiero de las instituciones financieras, con procedimientos fundamentales en la adopción de nuevas tecnologías. El análisis estadístico de las respuestas de los encuestados produjo promedios de 3.92 y 3.9 para las variables de los Sistemas Financieros y las Estrategias de Innovación Tecnológica, respectivamente, lo que determinaron que cumplen con altos niveles de importancia y juegan roles fundamentales en la gestión para el éxito de las compañías financieras de este tipo.

Los productos o servicios para desarrollar algunos están de acuerdo con la demanda del mercado, pero hay algunos que no por eso se genera las perdidas, o a veces son productos nuevos y tienen que esperar bastante tiempo para la adaptación y aceptación.

El tiempo adecuado para el lanzamiento de productos o renovación es letal, porque hay productos buenos, pero no son lanzados en momento ideal, y por último y lo más importante la capacitación del personal para la innovación porque de ellos depende el éxito de la empresa y la innovación interna y externa.

\section{DISCUSIÓN}

Las tecnologías emergentes están creando modelos y diseños comerciales para generar competencia y lo están logrando con la ayudad de la innovación disruptiva. Pisano (2015) la innovación incremental domina el arte de la innovación del modelo de negocio. Optando por la innovación tecnológica y la inversión en el modelo de negocio. De acuerdo con Jiménez \& Valle (2012) las estrategias de desarrollo de nuevos productos más innovadoras presentan mayor riesgo, incertidumbre y niveles de inversión en comparación con estrategias que persiguen la imitación.

Esto se debe a que este tipo de estrategias permiten desarrollar innovaciones de tipo incremental, altamente relacionados con el éxito de los nuevos productos conforme a los resultados de este estudio. 
Una estrategia de innovación ya sea de un proceso, producto o servicio, es introducirlo en el momento adecuado. El tiempo es un factor decisivo en la innovación, porque hay buenas ideas que llegaron en el momento que el mercado no estaba preparado y no funcionó. Hay innovaciones que empezaron a tiempo y se convirtieron en disruptivos.

Las empresas como Uber, Coworking vieron la necesidad y emprendieron, pero tener en cuenta que el que inicia algo nuevo tiene barreras para llegar al mercado, por las etapas de adaptación y aceptación.

Lo contrario pasa con la innovación incremental que ya es un producto existente y solo hacen mejoras como el IPhone y el Samsung, lo cual la innovación es más segura; para la innovación, la recomendación es la preparación y anticipación, conocimiento del entorno, la sociedad y las tendencias.

Según (Kim et al., 2017) los resultados que pudo observar es que se necesita conocer e implementar más tipos de innovación y debe ser de acuerdo a los objetivos planteados para alcanzar el éxito. Para Padayachee et al., (2017) la innovación disruptiva muestra una tecnología ágil para el cambio constante del mundo, que resuelve problemas específicos, convirtiéndose en un proyecto líder. Según Pisano (2015) las empresas tienen la posibilidad de elegir qué parte de sus esfuerzos para centrarse en la innovación tecnológica y la cantidad a invertir en la innovación del modelo de negocio.

Para Hacklin, Björkdahl, \& Wallin (2018) su objetivo principal fue investigar cómo se desarrolla la innovación del modelo de negocio en entornos dinámicos donde el valor está migrando rápidamente entre las empresas. Por lo que hizo tres observaciones principales.

El primero, pivotar el modelo comercial primario mejora la creación y captura de valor a un ritmo mayor que el lanzamiento de nuevos modelos comerciales secundarios. El segundo es que el pivote proactivo del modelo comercial primario genere una creación y captura de valor adecuadas que los cambios reactivos al modelo comercial primario. El tercero, bajo menores grados de migración de valor, el lanzamiento de modelos de negocios secundarios puede muy bien sostener o incluso mejorar la creación y captura de valor.

Por lo tanto, el trabajo identifica un desafío crítico: la necesidad de equilibrar los riesgos y las recompensas asociados con los modelos comerciales primarios y secundarios. Finalmente, se aconseja a los gerentes que evalúen las oportunidades asociadas con la migración de valor, correctamente cronometrado y administrado. (Hacklin et al., 2018) 


\section{CONCLUSIONES}

La innovación es clave para generar valor e impulsar a la empresa hacia el éxito, para esto se debe hacer con la innovación tecnológica y no tecnológica, lo cual va a depender mucho del tipo de empresa, además visualizar que tipo de estrategias de innovación utilizar en el área requerida y el tiempo adecuado. La innovación debe empezar por el área gerencial y servir como modelo para las demás áreas. Esta investigación sobre innovación en las empresas hará un aporte al conocimiento de la ciencia y generará reflexión de su importancia. Si las empresas no han empleado ninguna estrategia de innovación, puede que terminen en la quiebra, ya que se deben realizar cambios constantes y trabajar con objetivos, buscando siempre estrategias innovadoras, como la innovación abierta, innovación disruptiva, innovación incremental.

Las empresas tienen que tomar la decisión para innovar en el tiempo con la información adecuada para saber a dónde se está enfocando y con la oferta y demanda del mercado, ya que depende de eso el éxito que va a tener, además de promover la innovación continua. Según las investigaciones hay empresas que innovan en el proceso, en el producto o servicio y depende mucho del rubro de la empresa, además se puede concluir que las estrategias innovadoras son más relevantes que las estrategias de imitación. Las empresas necesitan determinar cuánto están gastando en la innovación, el análisis de Costo - beneficio, es fundamental para tomar la decisión de innovar o no innovar. Los costos implican una serie de recursos necesarios que se emplearán en el proyecto y son medidos en términos monetarios y tienen etapas o actividades (recursos humanos, financieros y productos)

Las empresas jóvenes respaldadas por empresas con fundadores que tienen una gran influencia (conocimiento o propiedad de la empresa) tienen más probabilidades de formar alianzas centradas en la innovación y formar alianzas exploratorias, destacando el poder de influencia de los fundadores. Con la finalidad de volver más sólidas sus iniciativas, permitiendo generar valor para el mercado y establecerse adecuadamente en el mercado objeto.

Con base a lo expresado se puede evidenciar la importancia que tienen las estrategias de innovación al interior de las organizaciones, ya que estas son el núcleo de desarrollo hacia la competitividad en su giro de trabajo, por tal razón considerar la inversión en equipos y la capacitación en áreas de la organización beneficiaran a largo plazo a las empresas, porque esto se volverá una cultura de creatividad e innovación constante. 


\section{REFERENCIAS BIBLIOGRÁFICAS}

Bogers, M., Chesbrough, H., \& Moedas, C. (2018). Open innovation: Research, practices, and policies. California Management Review, 60(2), 5-16. https://doi.org/10.1177/0008125617745086

Campos V, \& Sanchis J. (2015). Factores clave en el éxito de las empresas agrarias: El caso de las cooperativas hortofrutícolas en España. Tendencias, 2(2), 174-191. https://doi.org/https://doi.org/10.22267/rtend.151602.26

Chirinos, A. (2016). Revista Multidisciplinaria de Humanidades, Educación, Ciencia y Tecnología. Años II (Vol. 2). https://doi.org/10.35381/CM.V2I2.55

Clayton M. (1997). The innovator's dilemma : when new technologies cause great firms to fail. In HBSP (p. 252). Harvard Business School Press.

Fernández, M., \& Peña, I. (2009). Estrategia de innovación como factor determinante del éxito de las cooperativas vitivinícolas de Castilla La Mancha. Revesco. https://doi.org/https://dx.doi.org/10.5209/REVE

Franco, F., Mero, J., \& Mendoza, E. (2018). Strategies and innovation in family businesses. Revista Científica Ciencia Tecnología, 19, 12-23. Retrieved from http://cienciaytecnologia.uteg.edu.xn-ecestrategiaseinnovacinenlasempresasfamiliares-cpe

Hacklin, F., Björkdahl, J., \& Wallin, M. W. (2018). Strategies for business model innovation: How firms reel in migrating value. Long Range Planning, 51(1), 82-110. https://doi.org/10.1016/j.Irp.2017.06.009

Jiménez, D. J., \& Valle, R. S. (2012). Efectos de la estrategia de innovació $n$ en el é xito de los nuevos productos: El papel moderador del entorno. Revista Europea de Direccion y Economia de La Empresa, 21(4), 323-332. https://doi.org/10.1016/j.redee.2012.07.005

Karlsson, C., \& Tavassoli, S. (2016). Innovation strategies of firms: What strategies and why? Journal of Technology Transfer, 41(6), 1483-1506. https://doi.org/10.1007/s10961015-9453-4

Kim, C., Ondracek, J., Koseoglu, M. A., \& Barca, M. (2017). How do information technology companies use innovation to compete? Evidence from the USA and South Korea. International Journal of Business Innovation and Research, 12(3), 277-293. https://doi.org/10.1504/IJBIR.2017.10002765 
Martínez Andrés, \& Gelves Angy. (2017). La innovación inversa como estrategia de internacionalización para las empresas santandereanas (p. 11). Colombia: Gricani. Retrieved from http://ciani.bucaramanga.upb.edu.co/wpcontent/uploads/2017/10/Bryan-Martinez.pdf

Muigai, R. G., \& Gitau, S. N. (2018). Effect of innovation strategies on financial performance of the banking industry in kenya. European Journal of Economic and Financial Research, 3(0). https://doi.org/10.5281/zenodo.1291642

Naranjo, J. C., Jiménez, D. J., \& Sanz, R. (2012). ¿Es la cultura organizativa un determinante de la innovación en la empresa? Cuadernos de Economia y Direccion de La Empresa, 15(2), 63-72. https://doi.org/10.1016/j.cede.2011.07.004

OECD/Eurostat. (2018a). Oslo Manual 2018, Guidelines for collecting, reporting and using data on innovation. OECD. https://doi.org/10.1787/9789264065659-es

OECD/Eurostat. (2018b). Oslo Manual 2018: Guidelines for Collecting, Reporting and Using Data on Innovation. Paris: OECD. https://doi.org/10.1787/9789264304604-en

Oliver, R., \& Stezano, F. (2017). Innovación como campo de estudio en Latinoamérica 20102017: Brasil, Chile y México. Technol. Manag. Innov, 12(4), 70. https://doi.org/10.4067/S0718-27242017000400008

Padayachee, R., Matthee, M., \& Merwe, A. (2017). Disruptive technologies and IT decision making in an agile business environment. IEEE Africon, 6. https://doi.org/10.1109/AFRCON.2017.8095592

Pisano G. (2014). In Defense of Routine Innovation. Harvard Business Review. Retrieved from https://hbr.org/2014/06/in-defense-of-routine-innovation

Pisano G. (2015). You Need an Innovation Strategy. Harvard Business Review. Retrieved from https://hbr.org/2015/06/you-need-an-innovation-strategy

Rajapathirana, R. P. J., \& Hui, Y. (2018). Relationship between innovation capability, innovation type, and firm performance. Journal of Innovation and Knowledge, 3(1), 4455. https://doi.org/10.1016/j.jik.2017.06.002

Rocca, E. E., García, P. de L. D., \& Duréndez, G. G. A. (2016). Factores determinantes del éxito competitivo en la mipyme: un estudio empírico en empresas peruanas. $\begin{array}{llll}\text { Contabilidad } \quad y & \text { Negocios, } & \text { 52-68. }\end{array}$ https://doi.org/10.18800/contabilidad.201602.004 
Schumpeter, J. 1934 (2008). (2012). The Theory of Economic Development: An Inquiry into Profits, Capital, Credit, Interest and the Business Cycle. Journal of Comparative Research in Anthropology and Sociology, 3, 12. Retrieved from http://compaso.eu

Solano U, Martelo R, \& Acevedo D. (2018). Strategies of technological innovation in the financial system. Contemporary Engineering Sciences, 11(18), 861-869. https://doi.org/10.12988/ces.2018.8378 